\title{
Research Status and Development Trend of Internet of Things Technology
}

\author{
Qian Yan ${ }^{1,2 a}$ \\ ${ }^{1}$ Northeast Normal University, Computer Science and Information Technology \\ College ,Changchun, Jilin \\ ${ }^{2}$ Baicheng Normal College, Media Institute ,Baicheng ,Jilin \\ a qianyan1982@126.com
}

Keywords: The Internet of things; RFID; Sensors; IPv6

\begin{abstract}
In this paper, the current situation of research on Internet of things technology to carry on the analysis, the RFID technology, sensor network and detection technology, intelligent technology, nanotechnology, address of the IPv6 technology are summarized, analysis of existing problems in the development of the Internet of things, the development and application of the Internet of things technology is prospected.
\end{abstract}

\section{The Technology of the Internet of Things}

The key technology of things including RFID technology, sensor network and detection technology, intelligent technology, nanotechnology and the address of the TPv6 Technology.

A. RFID Technology

Radio frequency identification is a non-contact automatic identification technology, RF signal through its automatic target recognition and access to relevant data, identification process without human intervention, can work in a variety of harsh environment RFID technology can identify the high-speed moving objects and can also identify multiple tags, fast and convenient operation of RFID technology and the Internet, communication technology combined, can realize sharing items tracking and global information[1].

Agricultural technical difficulties and problems of RFID and now the following four aspects: the RFID anti collision prevention and conflict; study of the RFID antenna; the choice of operating frequency; security and privacy issues.

B. Sensor Network And Detection Technology

Sensor is the machine perception of the material world "sensory organs", can sense heat, force, light, electric, acoustic, displacement signal, is the network system processing, transmission, analysis and feedback to provide the most original information with small fault the development level of science and technology, the traditional sensor is gradually miniaturization, intelligent, informatization, network, the realization of the development of traditional sensor -- intelligent sensor embedded web sensor[2].

At present, research on sensor network technology for Internet of things including: the advanced testing technology and measurement and control network. the research of intelligent sensor network node. the study of the structure and the underlying protocol for sensor network organization.detection and control for the sensor network itself .sensor network security problem.

C. Intelligent Technology

Intelligent technology to effectively achieve some intended purpose, using a variety of methods and means adopted by implanting knowledge of intelligent system in the object, can make the object with each set of intelligence, and the user can realize the active or passive communication, is the key technology of the Internet of things[3].

Artificial intelligence theory research: formal method of intelligent information acquisition; the theory and method of massive information processing; development and utilization method of information under network environment; machine learning.

Advanced technology of human-computer interaction and the system sound, graphics, images, 
text and language $\mathrm{C}$ treatment; virtual reality technology and system; multimedia technology.

Intelligent control technology and system: the Internet of things is to endow the smart objects, can realize the human and the object of communication and dialogue, or even to achieve mutual communication and dialogue objects. In order to realize this goal, must be on the intelligent control technology and system implementation study. For example, research on how to control the intelligent service robot to accomplish a given task.

Intelligent signal processing: information of feature recognition and fusion technology, geophysical signal processing and recognition.

D. Nanometer Technology

Properties and applications of nano technology, is the study of the structure and size, in the 0.1-100nm van Itl materials mainly include: nano system physics, nano chemistry, nano material science, nano biology, Nano Electronics and nano processing science, nanomechanics these 7 relatively independent and mutual penetration of subjects and nano materials. The 3 nano devices. A study on the field of detection and characterization of nano scale. The preparation of nano materials and nano technology research is the foundation which, nano physics and nano chemistry is the theoretical basis of nanotechnology, nano electric and $\mathrm{R}$ is the most important content of Nanotechnology[4].

Using sensor technologies can detect the physical state, embedded intelligent objects can enhance network power through the transfer of information processing ability in the edge of the network, and nanotechnology advantage means that the things which the volume is more and more small objects to interact and connect.

The current trend of electrical $\mathrm{R}$ technology requirements of the device and system is smaller, faster, more cold. Refers to the smaller, fast response speed and more cold refers to power a single device to be small but smaller is not without limits of nanotechnology is the last frontier builders, its effects will be nano electric $\mathrm{R}$ giant, which are based on the quantum effect of nano $\mathrm{R}$ device electrically, nanostructured optoelectronic properties, nano electronic materials characterization well-atomic manipulation and atomic assembly etc..

E. IPv6 address technology

With the development of the IPv6 network closely, because each object networking connection will need IP address as the identification code, and the current IPv4 address has not enough. IPv6 owns the address space, its address space can meet the needs of node identification. At the same time, IPv6 uses a stateless address allocation scheme to solve the high efficiency of mass address allocation problem, after using a stateless address assignment, network is no longer need to save the address of node state maintenance update cycle address. This greatly simplifies the process of address allocation. The network can be achieved mass addresses assigned to very low resource consumption purposes[5].

Overall, IPv6 has a lot of suitable for Internet of things people scale application characteristics, not only to meet the networking address needs, while still meeting the IOT of node mobility and node redundancy, based on stream to guarantee quality of service demand, largely become the foundation of network technology of Internet of things applications.

\section{Difficulties and Problems}

\section{A. The Lack Of The Institution}

At present, the theory research of relative development is higher, but the practical application of Internet of things need to advance slowly, automatic control, information sensing, RFID, wireless communication and computer technology, the research of the Internet of things will bring the whole industry chain or to jointly promote the development of the industrial chain. Our country should as soon as possible in order to promote the introduction of relevant standards, the application process technology. At the same time, we should further in the technical, institutional and legal aspects of the perfect, so as to strengthen the supervision on the use of physical network.

\section{B. Information Security Guarantee}

Radio frequency identification is a key technology in the Internet of things is very important, it 
may lead to the issue of information security: the basic function of radio frequency identification tag to ensure that an arbitrary labeling, identification codes can be arbitrary scanning in the distance, and the label automatically, without distinction in response to reader's instructions, and will the transmission of information being stored to the reader. This characteristic can be used to track and locate a specific user or item, to obtain relevant information. The Internet of things to improve the efficiency and transparency through real-time data exchange, but also will be a personal preference data, what's more reflect the heart needs data exposed. But for the individual, do not know to master these data will take these data do, simply can not ensure that these data are not leak. Can not ensure that these data are not used to their disadvantage place, then will under the force from manufacturers, retailers, surveillance escape marketer etc.. Of course, government and law enforcement agencies can bring the technology for monitoring the citizen action. In the time of Internet of things, the daily management of the basic will be artificial intelligence processing, in order to reap benefits from the Internet of things virus people would even with the internet. Once the virus, it will likely result in factory production, the chaos of social order, and even directly threaten people's safety of life.

\section{The Influence Of Serious Pollution And Energy Consumption}

At present, in the Internet world, a large number of information processing computing capacity in consumption seemingly inexhaustible meaningless, consume a large amount of electric energy, too much carbon dioxide. By the middle of this century, compared with the existing Internet 'manufacturing "more information, the need for massive data calculation and processing therefore, in the future of the Internet of things, the need for timely collection and flow of good planning, good control of information, but also to distinguish and screening of these data make clear what study the competition; the data is useful. Even this is useless. Or where this is critical.

\section{Application Of Multi Field}

Specific for the Internet of things in different applications, requires research and development institutions should according to their respective field of application and understanding of the market demand, the objective setting of Internet of things applications, scientifically setting goals and content networking research and development, reasonable deployment of networking research and development resources, must not the sensor network academic general discussion as soon brought human ceremony will giant change of Internet of things, to realize the function of specific fields of the Internet of things the government decision-making and allocation of misleading limited research resources, such as automotive electronics field of Internet of things should be different from the medical and health; different applications have different requirement of network applications and services the quality requirements, the IOT nodes mostly resource constrained nodes, only through the special network technology can meet the application requirements of the Internet of things. For example: the Internet of things home application system is a try of China Telecom in the aspects of the Internet of things.

\section{The Development Trend}

China IOT standard established a joint working group, network related standards for the integration of domestic resources, research on joint industry parties to carry out the Internet of things technology of Internet of things, and actively promote the standardization work, accelerate the development of the Internet of things technology standard of our country meet the development needs of the government departments, for development decision network industry to provide comprehensive technical and standardized service support.

Many domestic and foreign university and carried out a lot of work in wireless sensor network side. In the very low power wireless sensor network, a self-organizing sensor network, mobile ad hoc network protocol, sensor network system application layer design, IP based mobile network and the self-organizing network combined with wireless sensor network technology and other aspects of research, in addition to universities and research institutes, the global each big famous enterprises has participated in the research of wireless sensor network. Chinese Unicom and Wuxi City Hall pole department in Nanjing "content network integration and application development of strategic 
cooperation framework agreement", the two sides will technology RFID, in IOT application research and related information services to carry out comprehensive cooperation. According to the agreement will be in China Unicom wired, wireless broadband network and the $3 \mathrm{G}$ network and the 'Internet of things "fusion to carry out a full range of cooperation, including: to jointly promote the sensor network technology standard research and industry research, sensor technology to promote the development and promotion of standards and application, research and development to promote the sensor network applications and services platform planning and sensor network study on the application of the industry, and the sensor network and the existing public network combined operation.

The Internet of things need automatic control, information sensing, RFID, wireless communication and computer technology, common development of the Internet of things will bring the whole industry chain and promote the industry chain can be predicted. After the 'Internet of things "in popularity. For animal. Ten straight and machine. The number of the sensor and the electronic tag and the interface device matching items will greatly exceed the mobile phone. According to the current demand for Internet of things, in recent years, according to the needs of billions of sensors and electronic benchmark, which will greatly promote the information technology component production, at the same time, increase the number of employment opportunities. To really establish effective Internet of things, there are two important factors: one is the scale, only with the scale, to make the items of intelligence play a role. Two is mobility, items are usually not static, but in the state of the movement, must keep the items in the state of motion, and even high-speed motion state can at any time for dialogue. China's wireless communications network covering both urban and rural areas, from the bustling city to remote rural areas, from Hainan Island to Mount Qomolangma, and everywhere the wireless network coverage. Wireless network infrastructure is essential to realize networking, digital signal electronic medium placed in animal, plant, machinery and goods produced by transmission through the wireless network whenever and wherever possible the ubiquitous out. Application of "cloud computing" technology, the real-time dynamic management of hundreds of millions of items becomes possible, make the network truly become a computer[6].

The Internet of things in itself, development represents the next generation of information technology, with the joint efforts of all aspects. The technology of the Internet of things will actively promote the whole economy of Chinese.

\section{Conclusion}

At present, the development of the Internet of things is at a stage of rapid development, theoretical research basically has gradually mature, but the specific application, still faces the standard is not clear, difficult to ensure product safety technology, low maturity, high cost and so on many restricting factors to promote industrialization and informationization background of the integration of the two in the national manpower, along with the formulation the national strategy, the investment of the enterprise, will help to solve these problems, the Internet of things as soon as possible to fully applied to every aspect of social life will no longer out of reach.

\section{References}

[1] Zhu Zhiting, Jiang Changhua. Education cloud open pan in the door of learning (J). Chinese education network, 2011, (6).

[2] Gong Li. Application of cloud computing in university education analysis (J). Journal of Yunyang Teachers'College, 2009, 29, (6): 97-98.

[3] China computer education. To simplify the education into the cloud (Eb/ol). Http://www.cce.com.cn/news/190741.html.

[4] the English Yi. The development of cloud computing and its influence on Education (J). 
Contemporary education forum, 2011, (1).

[5] Qin Ruxiang. The concept, characteristics and purpose of $\mathrm{J}$ education informationization. Theoretical exploration, 2004 (3): 63

[6] Chen Zhili emphasis on capacity building as the core in the international promotion of Chinese working conference, actively push into the Chinese language international promotion work DB/OL http: / /www. fmprc. Gov. cn/ce/cero/chn / jyxx / t261475. htm. CHN / jyxx / t261475. htm. 